\title{
How Intralymphatic Immunotherapy Can Affect Quality of Life and Symptoms in Patients With Permanent Seasonal Allergic Rhinitis, a Quasi- Experimental Design
}

\section{Maryam Khoshkhui}

Mashhad University of Medical Sciences Ghaem Hospital

Farahzad Jabbari

Mashhad University of Medical Sciences Ghaem Hospital

Fateme Shafiee Zargar

Mashhad University of Medical Sciences Ghaem Hospital

Nasrin Sadat Motevalli Haghi

Mashhad University of Medical Sciences Ghaem Hospital

Nazila Ariaee ( $\nabla$ ariaeen931@mums.ac.ir)

Mashhad University of Medical Sciences https://orcid.org/0000-0001-9053-5110

\section{Research}

Keywords: Allergic rhinitis, Allergen-specific immunotherapy, Intralymphatic immunotherapy

Posted Date: December 14th, 2020

DOI: https://doi.org/10.21203/rs.3.rs-122405/v1

License: (c) (1) This work is licensed under a Creative Commons Attribution 4.0 International License.

Read Full License 


\section{Abstract}

\section{Background}

Allergen-specific immunotherapy is known as the only disease-modifying treatment for IgE-mediated allergic disorders. Intra lymphatic immunotherapy (ILIT) is a safe, effective, and time-saving alternative to subcutaneous immunotherapy (SCIT). This study aimed to evaluate the effects and safety of ILIT in patients with moderate to severe allergic rhinitis.

\section{Methods}

In this clinical trial, fifteen patients with moderate to severe allergic rhinitis between 18-65 years old received monthly intralymphatic inguinal injections of an active allergen (1000 SQ-U Salsola kali pollen). Clinical effects were assessed before and 4 weeks after treatment and at two consecutive pollination seasons and following non-pollination season in April.

\section{Results}

No moderate or severe reactions were recorded following the ILIT treatment. Lymph node enlargement, local itching/erythema were seen in $6.7 \%, 13.3 \%$ of total respectively one day after injection. Patients who received ILIT experienced a significant clinical improvement in self-recorded seasonal allergic symptoms after treatment, as compared to themselves before ILIT. Quality of life significantly got better based on the Mini RQLQ questionnaire.

\section{Conclusion}

Although this study is based on a limited number of patients, ILIT with Salsola-pollen extract appears to decrease symptoms of allergic rhinitis without causing any crucial complications.

This clinical trial study was recorded in the Iran Registry of Clinical Trials (IRCT20161206031256N2).

\section{Introduction}

Allergic rhinitis is chronic respiratory congestion with a high prevalence over recent decades [1]. The quality of patients' life can be affected through some important complications of this condition [2]. Persistent allergic rhinitis can be exacerbated by seasonal episodes [3].

Although there are some non-pharmacotherapeutic measures to reduce the allergens exposure in the patient's environment [4], usually medicines are used to effectively treat allergic rhinitis. There are different classifications for medicaments used in allergic rhinitis treatment [5].

Recently, allergenic Immunotherapy is concerned an effective treatment in allergic Rhinoconjunctivitis and allergen-induced exacerbating asthma in some cases of intolerant or non-responsive to previous treatments [6]. 
The increase of allergen extract doses during Immunotherapy can reduce some symptoms induced by allergens' exposure. Immunotherapy can improve the quality of life and enhancing long-term tolerance to allergens $[7,8]$.

It is necessary to confirm the allergic process through the cutaneous prick test or serum level of specialized IgE, before immunotherapy [9]. One of the most common ones is sublingual swallow immunotherapy[10]. Meanwhile, intralymphatic immunotherapy(ILIT) is a new alternative through injecting allergens to inguinal lymph nodes 3 monthly instead of conventional injection over 3-5 years [11]. Although there is an equal clinical improvement, the level of the systemic reaction is lower in comparison to the conventional immunotherapy [12].

Considering the above benefits of ILIT, we decided to evaluate this method and its side effects on patients with allergic rhinitis who were sensitive to Salsola kali allergen.

\section{Patients And Methods}

\section{Study Design}

This study is a Quasi-Experimental Design to evaluate the effectiveness of ILIT in 12 patients suffering from permanent allergic rhinitis with seasonal exacerbation based on the ARIA guideline (2). Our study was done over 2018- 2019 in Allergy Clinic, Ghaem Hospital as a tertiary referral center in Mashhad in the northeast of Iran. Notably, the pollination period for Salsola kali allergen in Iran in September and October[13]. Thus, ILIT with Salsola pollen extract was performed on our cases before the pollination season while monthly injections were done in 3 months May, June, and July 2017. With each injection, a 1/10 ratio of Salsola pollen concentrate (Greer Company, USA) was entered into an inguinal lymph node under the guide of sonography through the 25 -gauge needle by an expert radiologist and supervision of an immunologist.

\section{Patients selection}

Our inclusion criteria included 18-65 years old patients experiencing moderate to severe permanent allergic rhinitis with seasonal exacerbation from summer to autumn, who were symptomatic at least for 2 years and resistant to conventional treatment. Besides, they should have a positive prick test for Salsola kali as well as having the specific IgE. Salsola was selected since it was the most common aeroallergen in the aria[13]. To prevent biases, we chose our cases according to their similar history of medication usage as well as the same dose and medication forms (nasal corticosteroids, oral antihistamines, nasal washing). Surely, all the study process and probable side effects were explained to every patient. Thus, provided they were interested to take part in our study, considering their ethical rights and the pertinent consent taken, they were included in our study.

Our exclusion criteria including pregnancy, having any diseases apart from allergic rhinitis (particularly uncontrolled asthma with FEV1 less than $70 \%$, insulin-dependent diabetes mellitus, cardiovascular 
diseases, autoimmune disorders, malignancies, a recent history of anaphylaxis), using specific medications (specially ACEI, MAOI, systemic corticosteroids, immunosuppressing drugs), having an upper respiratory infection, patients who did not give a consent to be involved in our study. Two questionnaires were used in our study including SNOT-22 for clinical manifestations and mini RQLQ, for quality of life.

\section{Intervention}

In the beginning, at the first visit (April 2018), medical history, physical examination, drug history for antiallergic treatment were obtained from all patients meanwhile questionnaires were completed. Clinical symptoms based on SNOT-22 and quality of life-based on the mini RQLQ questioners were assessed.

During the second to fourth visits, Salsola concentrate was injected monthly in inguinal lymph nodes. To recognize the side effects, each patient was admitted to the allergy-immunology ward in our center for 24 hours, and was observed for local and systemic symptoms over exact cardiovascular monitoring. Then, regarding the Visual Analog Scale (VAS), all side effects after each injection are divided into 2 categories of early-onset and late-onset (after one day).

At the fifth visit, 4 weeks after the last injection in July, again physical examination was done for all patients. Clinical symptoms based on SNOT-22 and quality of life-based on the mini RQLQ questioners were assessed.

At the sixth visit in September 2018 at the pollination season, all the process repeated. Consequently, in September 2019(next pollination season) and afterward in April 2020 (two years after the first visit before ILIT) quality of life and clinical symptoms through mentioned questioners were evaluated. Ultimately, all these new data after the ILIT were compared with previous data before the ILIT all the processes are in table 1.

\section{Statistical Analysis}

From a statistical viewpoint, since this is a pilot and quasi-experimental study, regarding the first and second type of bias (alpha: 0.05, beta: 0.2), our final case numbers were considered as 15 patients. Graph-Pad prism software was used in our study. Kolmogorov-Smirnov test and Friedman test were used for statistical assessment. The T-test was also applied to compare VAS in different stages.

\section{Results}

\section{Demography}

Among 15 patients, 7 patients were male with $46.7 \%$ of the total while females' figures were $53.3 \%$. The mean age was 33.3 \pm 6.7. Side effects of intralymphatic injection of concentrated allergen were evaluated via VAS based on clinical observations and self-patient reports.

\section{Clinical Outcomes}


Among 15 patients' lymph node enlargement was reported in 3 injections (6.7\%) immediately, and 12 injections (26.7\%) one day after injection. Early-onset side effects at the injection site were revealed as local itching in 6 cases (13.3\%), erythema in 6 cases (13.3\%), angioedema/ urticaria in 3 cases of injections (6.7\%). The details can be seen in Table 2. The severity of pain had a mean value of $1.5 \pm 0.5$ based on the VAS. This value is compared with the pain severity induced intravenous line accessing.

\section{Clinical outcome}

Regarding the SNOT-22 questionnaire, nose, ear problems, sleep disorders, psychology, and activity were evaluated as before ILIT and mentioned dates after that, as can be seen in Table 3

Considering the pairwise test, we compared SNOT22 total scores during the period. This data has been shown in Fig2. There was a significant difference in scores between before and after ILIT $(p<0.001)$. Furthermore, a significant difference was revealed in these scores between before immunotherapy and the first pollination $(p<0.001)$. In contrast, we did not find any significant difference in SNOT22 total scores after immunotherapy and at the first pollination season $(p=0.08)$. Statistical analysis revealed although there were lower grades in SNOT22, there was a significant difference in two pollination seasons in Sep 2018 and Sep 2019. It means that ILIT was not much effective after one year, particularly in the pollination season. Comparing April 2018 before ILIT and 2020 after ILIT showed a significant difference $(p<0.001)$, which means patients had overall amelioration.

\section{Quality of life}

As for the Mini RQLQ questionnaire, quality of life was scored during five steps including before ILIT, 4 weeks after ILIT, first pollination season in the same year, next year pollination season, and following April. The mean value was reported for each step in Fig 3. It was notable that a significant difference was reported in quality of life between before ILIT in April and after that in Aug and at the first pollination season in September in the same year $(p<0.001)$. There was also a significant difference between the before ILIT in April and after ILIT in the next April $(p<0.001)$. There was a statically significant difference in the quality of life between the first pollination season and the second one in the next year $(p<0.001)$.

\section{Discussion}

As for SNOT-22 questionnaire contents, nose-ear-sinuses problems and sleep disorders were evaluated. There was a significant difference before and after immunotherapy, between before immunotherapy and the first pollination season. Considering the Mini RQLQ questionnaire, notably, a significant difference was reported in the quality of life between before and after treatment and also between before treatment and at the end of pollination. The serum level of IgE for Salsola was evaluated before and after the ILIT and also at the first pollination season. This rate had a significant difference.

Some side effects were revealed as lymph node enlargement, local itching, erythema, angioedema/ urticaria, the symptoms of which were seen during our study, while nasal, pulmonary, and abdominal 
symptoms were not appreciated in any of the cases. Pain at the injection site was completely tolerable regarding what the patients defined.

The first study for ILIT was done by Senti et al. They comprised ILIT in 58 cases versus subcutaneous immunotherapy in 54 cases by using grass pollen for the first time in 2008 [14].

Senti et al used ILIT over 3 injections to induce cat allergy tolerance in 2012 as a new idea [15]. Similarly, to us, we also used 3 monthly injections of ILIT to induce allergic rhinitis for Salsola.

Our results would be comprised of their findings considering the relevant conclusions. All these studies reported that ILIT had no moderate to severe side effects, as same as ours, all side effects were mild and were resolved without any treatment.

Lee et al evaluated 11 cases of intralymphatic immunotherapy via using cat and dog allergens in an open pilot study[16]. Exclusively, Lee and et al reported 2 cases of anaphylaxis and one case of severe local reaction but there was not any death report[17]. We found lymph node enlargement as an early and late side effect in $6.7 \%$ and $26.7 \%$ of total respectively, which continued for one week and revealed without any treatment.

Hylander et al in a double-blind study on 21 patients, performed Intralymphatic allergen-specific immunotherapy for Birch pollen \& grass pollen as a safe alternative treatment method for pollen-induced allergic rhinitis [16]. A detailed of our findings considering the side effects of this method has been prepared in table 2. As it is seen in table-2 there is not any anaphylaxis, and also none of our cases were excluded because of side effects.

Several years later, again, Hylander et al during the controlled trial on 20 Active patients vs. 15 placebo cases, applied Intralymphatic allergen-specific immunotherapy for Birch pollen \& grass pollen-induced rhinoconjunctivitis[18]. Based on the VAS, the pain score was defined from zero to ten. The mean value for pain score was $1.15 \pm 0.5$ in our study in contrast to 0.4 regarding Hylander's result[18]. While we used Salsola, using different allergen concentrate probably makes different pain severity.

As mentioned before, recently, most scientists believe that ILIT can improve quality of life and resolve the majority of allergic rhinoconjunctivitis problems. This idea has been proved in two studies using grass pollen by Patterson et al [19] on 8 patients and Lee.SP et al on 7 patients [17]. While our sample volume was more than them (15 patients), considering Mini RQLQ the mean value for the quality of life was improved from 46.2 before the ILIT to 6.5 and 18.3 at the end of ILIT and pollination respectively. As a similar concept, Lee.SP et al also reported an improvement in the quality of life from 71.2 before injections to 52.3 after injections[17].

Witten et al in a double-blinded study on 12 cases of ILIT showed a significant difference in Mini RQLQ as a result of ILLT by grass pollen[20]. As the same as our results, with a significant difference in Mini RQLQ, the quality of life got better in our study. It should be mentioned that the SNOT-22 questionnaire had not been used in any previous study and we creatively used this item for evaluating patients' satisfaction in a 
detailed view. We found a significant difference for each item between before and after immunotherapy, similarly, between before immunotherapy and at the pollination season. Our study highlighted two consecutive pollination seasons the first one after pollination season revealed an improvement and the next year was also patients experienced similar symptoms to pre ILIT. On the other hand, comparing to pre ILIT in first April, patients indicted alleviated symptoms in the next April. Comparing the second pollination season with the next April proved that ILIT can be helpful more in non-pollination seasons. These data were the same as quality of life as indicated in next April still ameliorating impacts of ILIT could be observed.

Although some immunologists recommend measuring IgG4, and specific IgE to assess the response of ILIT[16, 21], we couldn't do it according to our limitations.

In fact, because of our limited case number, it is suggested to design more studies with a larger sample volume on ILIT. On the other hand, we just focused on allergic rhinitis, while it is needed to evaluate ILIT as a safe and effective method for some diseases like asthma. This method does not need any premedication while safety, less and rare side effects, and also lower costs are interesting benefits that can motivate scientists to assess the ILIT long-term effectiveness on clinical improvement in a wide variety of allergic disorders which are resistant to conventional treatments.

\section{Conclusion}

In fact, because of our limited case numbers, it is suggested to design more studies with a larger sample volume on ILIT. On the other hand, we just focused on allergic rhinitis, while it is needed to evaluate ILIT as a safe and effective method for some diseases like asthma. This method does not need any premedication while safety, less and rare side effect, and also lower costs are interesting benefits that can motivate scientists to assess the ILIT long-term effectiveness on clinical improvement in a wide variety of allergic disorders which are resistant to conventional treatments.

\section{Declarations}

Ethics Committee Approval and Patient Consent:This experiment has been approved by the ethics committee of Mashhad University of Medical Science (IR.MUMS.fm.REC1395.574). Also, this clinical trial study was recorded in the Iran Registry of Clinical Trials (IRCT20161206031256N2). All patients signed a written informed consent agreement.

Consent of Publication: The authors has the licence of publication and mentione in the contraction of the grant that authors are responsible for thisand have to make data available to the public.

Availability of Data and Material: All data and questinaires is available on journal request

Compete of Interests:The authors have declared that no competing interest exists. 
Funding:This research was financially supported by the Allergy Research Center, Mashhad University of Medical Sciences, and vice president for research, Mashhad University of Medical Sciences, Mashhad, Iran (grant number: 951057).

Authors Contribution: Maryam Khoshkhui designe the study, Frarhzad Jabbari visit the patients and recruit them, Fatemeh Shafiee do radiology guid, Nazila Ariaee Preparing manuscript, data Analysis, help to study design. Nasrin Sadat Motavali Haghi visiting patients filling questinares.

Acknowledgments: We would like to deeply appreciate the unwavering efforts of the staff of the Immunology-Allergy department in Ghaem hospital in Mashhad.

\section{Abbreviations}

SNOT-22 TEST: Sino-Nasal-Outcome Test Questionnaire

Mini-RQLQ: Mini Rhino conjunctivitis Quality of Life Questionnaire

FEV-1: First second of Forced Expiration

IgE: Immunoglobin E

IgG: Immunoglobin G

EDTA: Ethylene Diamine Tetra Acetic Acid

MAOI: Mono Amine Oxidase Inhibitor

ACEI: Angiotensin-Converting Enzyme Inhibitor

ELISA: Enzyme-Linked Immunosorbent Assay

VAS: Visual Analogue Scale

ILIT: Intra Lymphatic Immunotherapy

\section{References}

1. Zuberbier T, Lötvall J, Simoens S, Subramanian S, Church MK. Economic burden of inadequate management of allergic diseases in the European Union: a GA2LEN review. Allergy. 2014;69(10):1275-9.

2. Brożek JL, Bousquet J, Baena-Cagnani CE, Bonini S, Canonica GW, Casale TB, et al. Allergic Rhinitis and its Impact on Asthma (ARIA) guidelines: 2010 revision. Journal of Allergy and Clinical Immunology. 2010;126(3):466-76. 
3. Alyasin S, Nabavizadeh SH, Houshmand H, Esmaeilzadeh H, Jelodar S, Amin R. Short time efficiency of rhinophototherapy in management of patients with allergic rhinitis resistant to medical therapy. Iranian Journal of Allergy, Asthma and Immunology. 2016;15(4):317-27.

4. Custovic A, Tovey E. Allergen control for prevention and management of allergic diseases. Middleton's Allergy: Elsevier; 2014. p. 1406-15.

5. Meltzer EO, Bensch GW, Storms WW, editors. New intranasal formulations for the treatment of allergic rhinitis. Allergy \& Asthma Proceedings; 2014.

6. Nelson HS. Injection immunotherapy for inhalant allergens. Middleton's Allergy: Elsevier; 2014. p. 1416-37.

7. Calderon MA, Alves B, Jacobson M, Hurwitz B, Sheikh A, Durham S. Allergen injection immunotherapy for seasonal allergic rhinitis. Cochrane database of systematic reviews. 2007(1).

8. Schmitt J, Schwarz K, Stadler E, Wüstenberg EG. Allergy immunotherapy for allergic rhinitis effectively prevents asthma: results from a large retrospective cohort study. Journal of Allergy and Clinical Immunology. 2015;136(6):1511-6.

9. von Moos S, Kündig TM, Senti G. Novel administration routes for allergen-specific immunotherapy: a review of intralymphatic and epicutaneous allergen-specific immunotherapy. Immunology and Allergy Clinics. 2011;31(2):391-406.

10. Hoseini R, Jabbari F, Rezaee A, Rafatpanah H, Yousefzadeh H, Ariaee N, et al. House dust mite sublingual-swallow immunotherapy in perennial rhinitis: a double-blind, placebo-controlled Iranian study. Journal of biological regulators and homeostatic agents. 2018;32(1):83-8.

11. Burks AW, Calderon MA, Casale T, Cox L, Demoly P, Jutel M, et al. Update on allergy immunotherapy: American academy of allergy, asthma \& immunology/European academy of allergy and clinical immunology/PRACTALL consensus report. Journal of Allergy and Clinical Immunology. 2013;131(5):1288-96. e3.

12. Petalas K, Durham S. Allergen immunotherapy for allergic rhinitis. Rhinology. 2013;51(2):99-110.

13. Oskouei YM, Hosseini RF, Ahanchian H, Jarahi L, Ariaee N, Azad FJ. Report of common aeroallergens among allergic patients in northeastern Iran. Iranian journal of otorhinolaryngology. 2017;29(91):89.

14. Senti G, Vavricka BMP, Erdmann I, Diaz MI, Markus R, McCormack SJ, et al. Intralymphatic allergen administration renders specific immunotherapy faster and safer: a randomized controlled trial. Proceedings of the National Academy of Sciences. 2008;105(46):17908-12.

15. Senti G, Crameri R, Kuster D, Johansen P, Martinez-Gomez JM, Graf N, et al. Intralymphatic immunotherapy for cat allergy induces tolerance after only 3 injections. Journal of Allergy and Clinical Immunology. 2012;129(5):1290-6.

16. Hylander T, Latif L, Petersson-Westin U, Cardell LO. Intralymphatic allergen-specific immunotherapy: an effective and safe alternative treatment route for pollen-induced allergic rhinitis. Journal of allergy and clinical immunology. 2013;131(2):412-20.

17. Lee SP, Choi SJ, Joe E, Lee SM, Lee MW, Shim JW, et al. A pilot study of intralymphatic immunotherapy for house dust mite, cat, and dog allergies. Allergy, asthma \& immunology research. 
2017;9(3):272-7.

18. Hylander T, Larsson O, Petersson-Westin U, Eriksson M, Georén SK, Winqvist O, et al. Intralymphatic immunotherapy of pollen-induced rhinoconjunctivitis: a double-blind placebo-controlled trial. Respiratory research. 2016;17(1):10.

19. Patterson AM, Bonny AE, Shiels WE, Erwin EA. Three-injection intralymphatic immunotherapy in adolescents and young adults with grass pollen rhinoconjunctivitis. Annals of Allergy, Asthma \& Immunology. 2016;116(2):168-70.

20. Witten M, Malling H-J, Blom L, Poulsen BC, Poulsen LK. Is intralymphatic immunotherapy ready for clinical use in patients with grass pollen allergy? Journal of allergy and clinical immunology. 2013;132(5):1248-52. e5.

21. Senti G, Kündig TM. Novel delivery routes for allergy immunotherapy: intralymphatic, epicutaneous, and intradermal. Immunology and Allergy Clinics. 2016;36(1):25-37.

\section{Tables}

Table 1. Details of the process of the investigation in consort table 


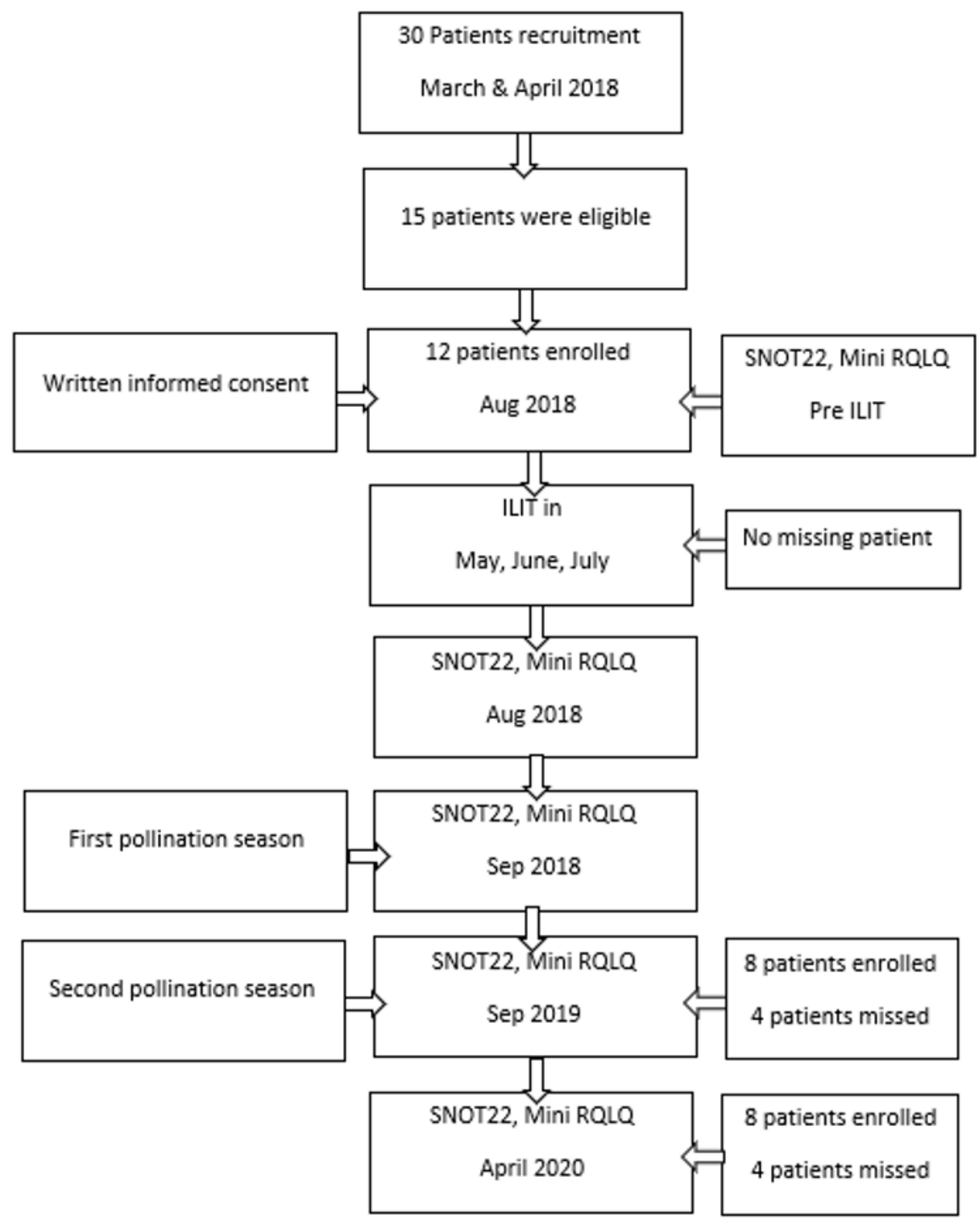

Table2. Incidence of side effects of ILIT in our study in a total of 45 injections. 


\begin{tabular}{|lll|}
\hline Side effects & $\begin{array}{l}\text { Early-onset } \\
\mathrm{N}(\%)\end{array}$ & $\begin{array}{l}\text { Late-onset } \\
\mathrm{N}(\%)\end{array}$ \\
\hline Lymph node enlargement & $3(6.7 \%)$ & $12(26.7 \%)$ \\
\hline Local itching & $6(13.3 \%)$ & - \\
\hline Local redness & - & - \\
\hline Angioedema/Urticaria & $3(6.7 \%)$ & - \\
\hline Nasal Symptoms & - & - \\
\hline Pulmonary Symptoms & - & - \\
\hline Abdominal Symptoms & - & - \\
\hline
\end{tabular}

Table 3. The mean score of patients' symptoms in three stages based on the SNOT-22 questionnaire

\section{Mean value Score \pm SD}

\section{Symptoms}

\begin{tabular}{cccc}
$\begin{array}{c}\text { Pre ILIT } \\
\text { (April) }\end{array}$ & $\begin{array}{c}\text { Post ILIT } \\
(\text { Aug })\end{array}$ & $\begin{array}{c}1^{\text {st }} \text { Pollination } \\
(\text { Sep })\end{array}$ & $\begin{array}{c}2^{\text {nd }} \text { Pollination } \\
(\text { Sep })\end{array}$ \\
\hline
\end{tabular}

$\begin{array}{llllll}\text { Nose } & 23.3 \pm 6 & 12.1 \pm 2.9 & 15.3 \pm 6 & 19 \pm 6.9 & 12.8 \pm 8\end{array}$

Ear

$3.3 \pm 1$

$1 \pm 0.1$

$1.5 \pm 0.1$

$3 \pm 3.3$

$1.8 \pm 1$

Sleep state

$9.26 \pm 1.9$

$2.1 \pm 0.2$

$0.8 \pm 0.3$

$10 \pm 4.2$

$8.4 \pm 4.7$

Activity

$6.13 \pm 3$

$1.5 \pm 0.1$

$0.5 \pm 0.2$

$8.5 \pm 7.5$

$4 \pm 2.3$

Psychology

$7.51 \pm 1.6$

$1.1 \pm 0.1$

$0.5 \pm 0.2$

$6.4 \pm 3.8$

$2 \pm 0.1$

\section{Figures}




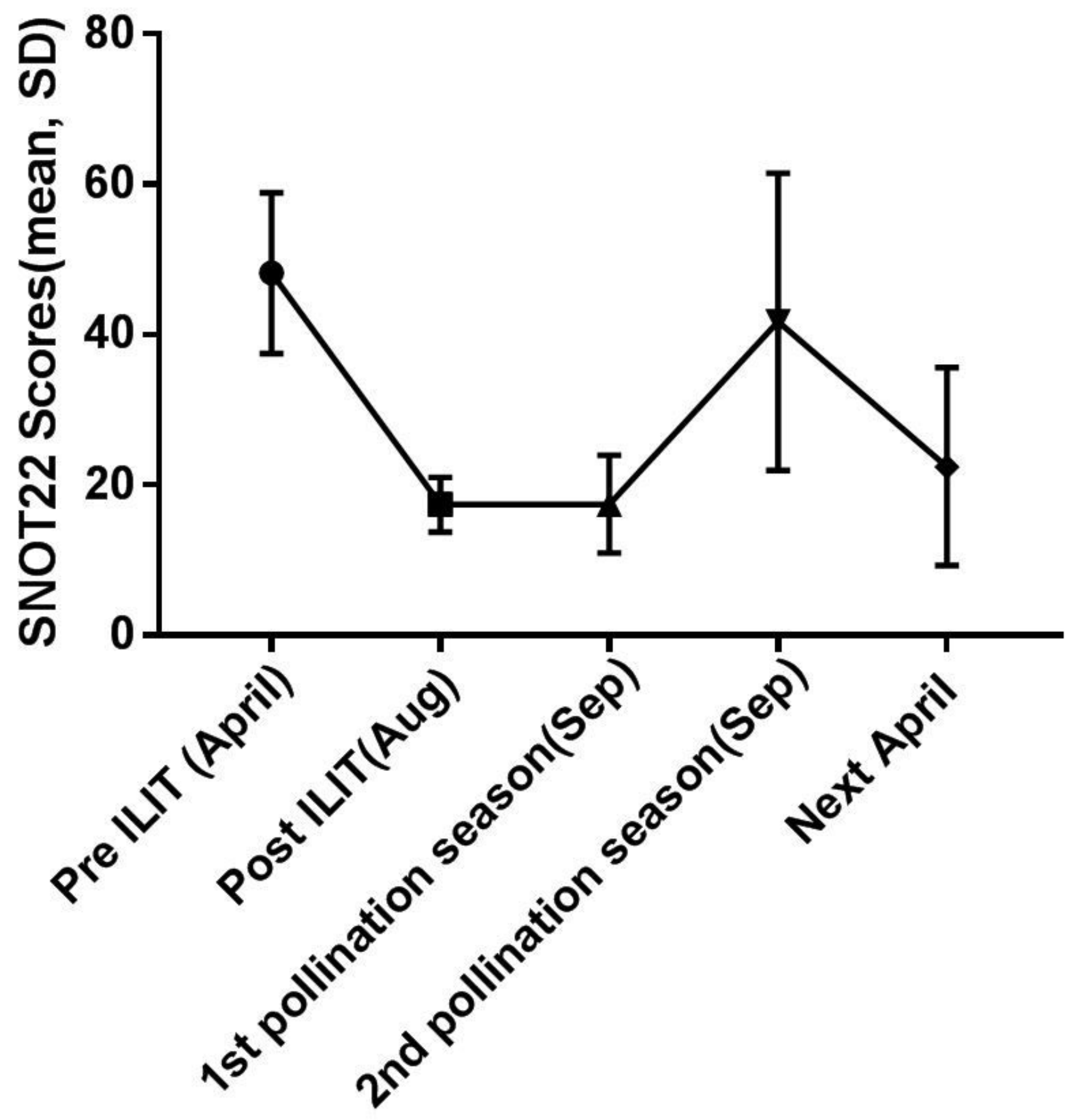

Figure 1

Mean score of SNOT22 questioner before ILIT, after ILIT, at the first pollination season, the second one, and next April. 


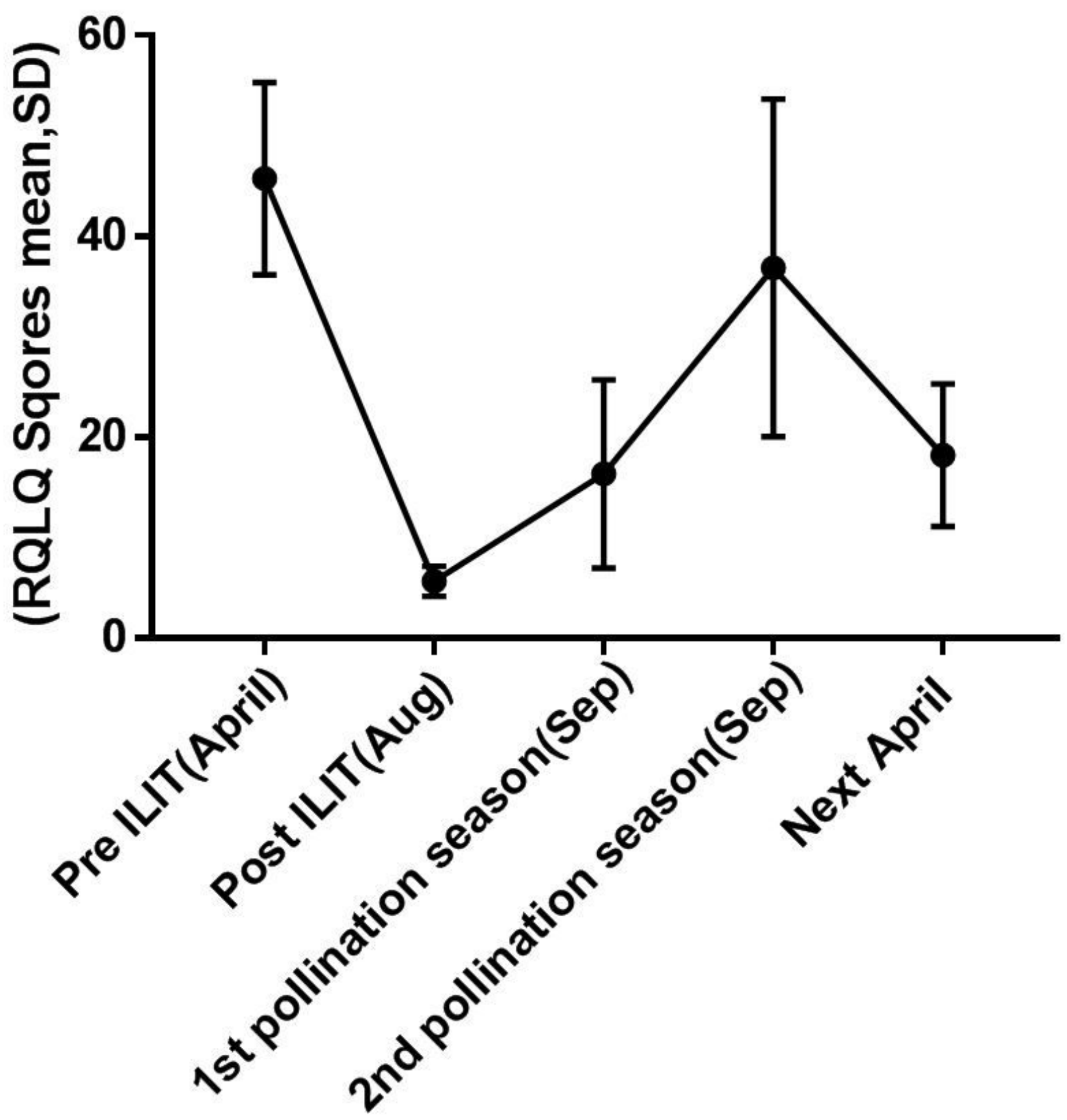

Figure 2

Quality of life (Mini-RQLO) before ILIT, after ILIT, at the first, the second pollination season, and the next April. 